\title{
Online Open Circuit Fault Diagnosis for Rail Transit Traction Converter Based on Object-Oriented Colored Petri Net Topology Reasoning
}

\author{
Lei Wang, Chunmei Xu, Lijun Diao, Jie Chen, Ruichang Qiu, and Peizhen Wang \\ School of Electrical Engineering, Beijing Jiaotong University, Beijing 100044, China \\ Correspondence should be addressed to Lei Wang; leiwang@bjtu.edu.cn
}

Received 13 May 2016; Accepted 31 August 2016

Academic Editor: Qingling Zhang

Copyright @ 2016 Lei Wang et al. This is an open access article distributed under the Creative Commons Attribution License, which permits unrestricted use, distribution, and reproduction in any medium, provided the original work is properly cited.

For online open circuit fault diagnosis of the traction converter in rail transit vehicles, conventional approaches depend heavily on component parameters and circuit layouts. For better universality and less parameter sensitivity during the diagnosis, this paper proposes a novel topology analysis approach to diagnose switching device open circuit failures. During the diagnosis, the topology is analyzed with fault reasoning mechanism, which is based on object-oriented Petri net (OOCPN). The OOCPN model takes in digitalized current inputs as fault signatures, and dynamical transitions between discrete switching states of a circuit with broken device are symbolized with the dynamical transitions of colored tokens in OOCPN. Such transitions simulate natural reasoning process of an expert's brain during diagnosis. The dependence on component parameters and on circuit layouts is finally eliminated by such circuit topology reasoning process. In the last part, the proposed online reasoning and diagnosis process is exemplified with the case of a certain switching device failure in the power circuit of traction converter.

\section{Introduction}

Switching device failures account for a large part of all the malfunctions in a converter-motor system [1]. In some cases of rail transit system, switching device failures may even be up to $25 \%$. In rail transit converters, the switching devices, which are adopted in power circuit, fall into two categories: the controllable ones and the uncontrollable ones. The controllable devices could be thyristors, GTOs, IGBTs, and so forth. Among all of them, IGBT has been commonly chosen in most applications. The uncontrollable device refers to diodes in all the cases. Among the failures of all possible switching devices, IGBT failures are much more than diode failures. IGBT failures mainly appear as shoot-throughs (short circuit after failure) or break-downs (open circuit after failure) between the collector and emitter terminals. Since it is much easier to detect shoot-through with IGBT trigger unit [2], in this paper we deal mainly with IGBT break-downs, that is, open circuit faults.

The possible cause of IGBT break-downs could be

(i) because of bond wire lift-offs inside an IGBT module; (ii) because IGBT die is burnt out by over-temperature that originates from lowered heat transfer property owing to degraded solder;

(iii) because of poor IGBT selection in changing seriously or over-range outputs.

When IGBT break-down happens in a power circuit, the fault diagnosis for malfunctioning device location should be carried out immediately to prevent further failures. Among all the diagnosis approaches that have been proposed, the diagnosis based on topology analysis has made itself important and convenient [3-5]. However, in topology-based approach, much more should be done. In [6,7], Shi and Shang et al. focus their work on the deduction of hybrid switching topology; in [8], Ma and Zhang come up with an identification approach by measuring a set of topology linearity irrelevant circuit state variables. They come up with only probable theoretical approaches, and there is some distance between their models and field applications. In [9], Zhang et al. identify topology with residual analysis, but such approach depends seriously on circuit parameters, so 
the effectiveness in application is somehow limited. To avoid such dilemma, this paper aims at proposing a novel topology analysis approach, which is less sensitive to circuit parameters and easier for field applications and shows more universality.

For a power circuit which consists of several switching devices, its operation between certain switching states could be seen as dynamical transition process between certain discrete events. Being an ideal tool to describe the concurrency, the conflict, and the causality among the internal components of a discrete system, Petri net shows more advantages in dealing with the dynamical process of such discrete events [10-12]. Moreover, the object-oriented colored Petri net (OOCPN), which is modified with colored token and more flexible transitions by us, has been made more efficient in power circuit topology identification. The dynamical transitions of colored token among places inside an OOCPN simulate human brain activities vividly, just like those activities that happen in the process of an expert's failure reasoning and malfunctioning device locating. Such universality makes OOCPN a useful tool in topology analysis and in fault diagnosis.

The elimination of the dependence on circuit layouts and especially on component parameters is the key problem in our work. By the application of OOCPN, such dependence is eliminated naturally with the movement of colored tokens, so that the reasoning process is completely not affected by such factors above.

\section{The Topology Reasoning Machine Based on OOCPN for Power Electronic Circuits}

For a power electronic circuit, the on or off state combinations of switching devices change in a discrete way. However, the currents and voltages in the circuit change continuously between two different on/off state combinations. Such case meets the characteristics of a so-called Dynamic System of Discrete Events (DSDE) perfectly. Different on/off state combinations result in different changing patterns of the analogue voltages and currents. Such patterns offer perfect signatures of corresponding on/off state combinations, which is also the case of power circuits with switching device failures. By analyzing the changing patterns of voltages and currents, a malfunctioning switching device could be recognized at the same time.

For a power electronic circuit which is equivalized to a network with $N$ inputs and $M$ outputs, its topology is shown in Figure 1.

In Figure 1, the input ports are defined to be $\mathrm{Bcl} \sim \mathrm{Bc} M$, with $M$ being the number of input ports. The output ports are $\operatorname{Brl} \sim \mathrm{BrN}$, with $N$ being the number of output ports. The component $E_{j i}$ corresponds to an IGBT or a diode or an IGBT + diode (in parallel or in series) branch that brigdes between the $i$ th input and the $j$ th output.

Based on graph theory, the switching circuit topology reasoning procedure carried out by an automatic reasoning machine is as follows:

(1) To derive possible ideal current and voltage signature set, on the basis of external characteristics of the

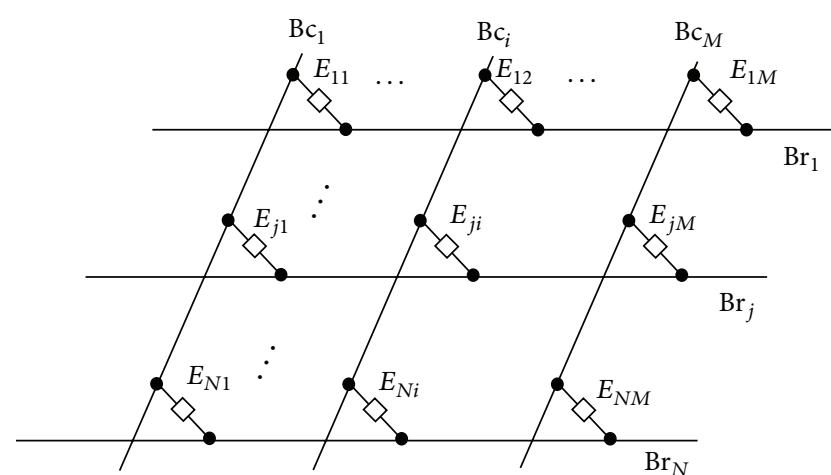

FIgURE 1: The equivalent network of a power electronic circuit with multi-input/output ports.

circuit (i.e., input and output waveforms or values of the circuit).

(2) To derive ideal current flow capability of each branch that is placed in the intersection between certain ports, by analyzing every single ideal current and voltage signature. Such capability could be given as unidirectional, bidirectional, and so forth.

(3) To derive actual current flow capability of each branch that is placed in the intersection between certain ports, by analyzing all the current and voltage signatures that have been actually detected and recorded.

(4) To carry out the reasoning process for switching branches that have failed to turn on, by analyzing the difference between the ideal and the actual current flow capabilities of each branch.

In order to meet the needs of the topology identification and reasoning, we propose a novel object-oriented colored Petri net (OOCPN) by introducing colored attribute and function attribute into conventional object-oriented Petri net (OOPN) [13-15]. The additionally introduced colored attribute and function attribute enable OOCPN to be better and more comprehensive in describing the inner structure of a switching branch. Based on the definition of colored Petri net [16], here the mathematical definition of OOCPN could be given as follows:

A multivariable model of $\{S, Y, P, T, A, N, C, G, E, I\}$ becomes an OOCPN, when

(1) $S$ is the color attribute space of OOCPN, where $S=\left\{\varsigma_{1}, \varsigma_{2}, \ldots, \varsigma_{m}\right\}$ ( $\varsigma_{i}$ corresponds to the $i$ th color attribute set of a token in OOCPN, and $m$ is the number of member variables);

(2) $Y$ is member method of a token, where $Y: S \rightarrow \varsigma_{i}$, $\varsigma_{i} \in S$

(3) $P$ is the place set of OOCPN, where $P=\left\{p_{1}, p_{2}\right.$, $\left.\ldots, p_{n}\right\}$ ( $p_{i}$ is the $i$ th place; $n$ is the number of places);

(4) $T$ is the transition set of OOCPN, where $T=\left\{t_{1}, t_{2}\right.$, $\left.\ldots, t_{o}\right\}\left(t_{i}\right.$ is the $i$ th transition; $o$ is the number of transitions); 


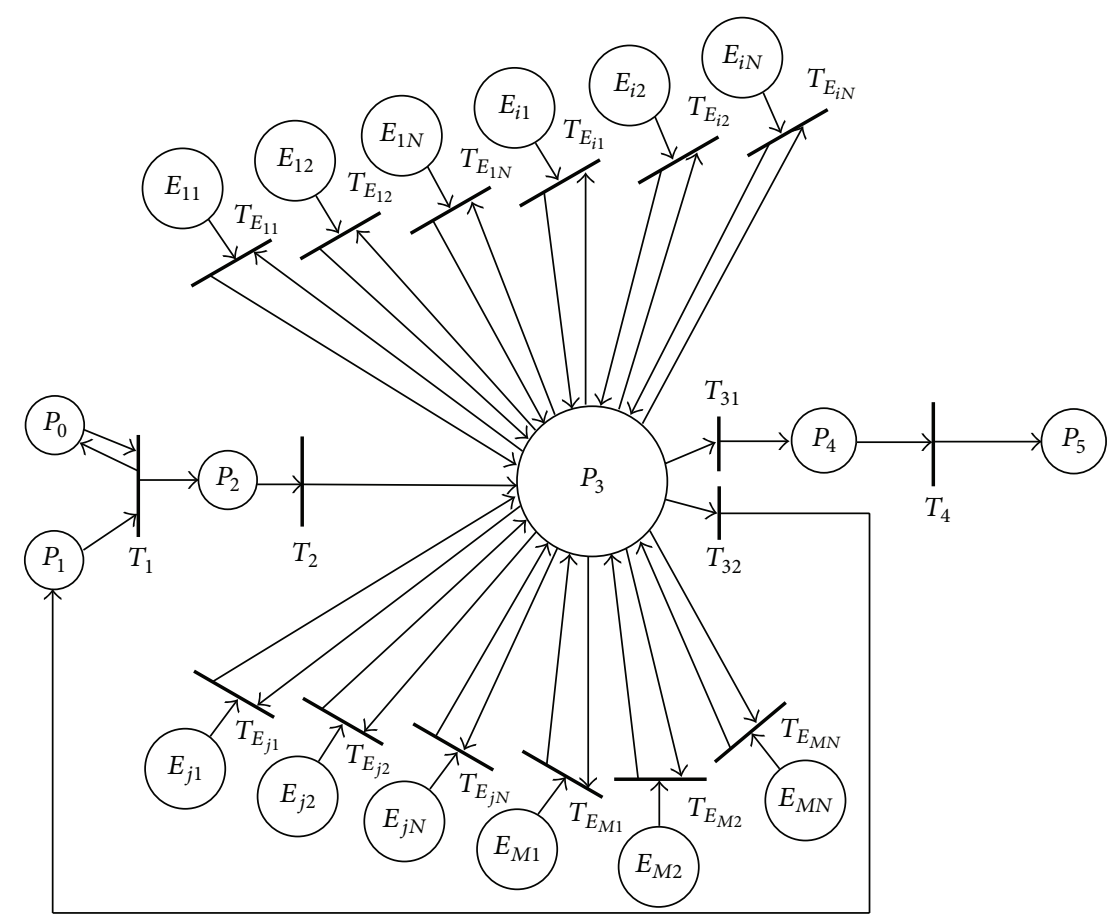

FIGURE 2: The OOCPN reasoning model for topology analysis.

(5) $A$ is the directed arc set of OOCPN, where $A=\left\{a_{1}, a_{2}\right.$, $\left.\ldots, a_{k}\right\}\left(a_{i}\right.$ is the $i$ th directed arc; $k$ is the number of directed arcs);

(6) $P \cap T=P \cap A=T \cap A=\Phi$;

(7) $N$ is the node function of OOCPN, where $N: A \rightarrow$ $P \times T \cup T \times P$

(8) $C$ is the color function of OOCPN, where $C: P \rightarrow S$;
(9) $G$ is the escorting function of OOCPN, where $G$ : $T \rightarrow f(T)$. When $G\left(t_{i}\right)$ is the escort function of $t_{i}$, it gives

$\forall t_{i} \in T:$

$\left[\operatorname{Type}\left(G\left(t_{i}\right)\right)=\right.$ BOOL $\wedge$ Type $\left.\left(\operatorname{Var}\left(G\left(t_{i}\right)\right)\right) \subseteq \Theta, \Theta=\bigcup_{j=1}^{m} \varsigma_{j}\right] ;$

(10) $E$ is the arc-expression function of OOCPN, where $E$ : $A \rightarrow h(A)$, and if $p\left(a_{i}\right)$ is $N\left(a_{i}\right)$ 's place,

$$
\forall a_{i} \in A: \quad\left[\operatorname{Type}\left(E\left(a_{i}\right)\right)=C\left(p\left(a_{i}\right)\right) \wedge \operatorname{Type}\left(\operatorname{Var}\left(E\left(a_{i}\right)\right)\right) \subseteq \Theta, \Theta=\bigcup_{j=1}^{m} \varsigma_{j}\right]
$$

(11) $I$ is the initialization function of OOCPN, where

$$
\forall p_{i} \in P: \quad\left[\text { Type }\left(I\left(p_{i}\right)\right)=C\left(p_{i}\right)_{\mathrm{MS}}\right]
$$

In [17], a CPN model is adopted for the location of acting breakers in a power grid with short circuit faults. Here we improve and revise it into an OOCPN model and adopt the revised OOCPN model into topology analysis field. Such improved model forms a human-like reasoning machine and is shown in Figure 2.

In the OOCPN reasoning machine, we define its specific colored token as $\{\mathrm{Cy}, \mathrm{Cc}, \mathrm{Cp}, \mathrm{Ca}, \mathrm{Cn}, \mathrm{Dr}, \mathrm{Fun}\}$, where $\mathrm{Cy}$ is the intersection color (or attribute) of a certain switching branch; $\mathrm{Cc}$ is the current flow color of the branch; $\mathrm{Cp}$ is the reference current signature color; $\mathrm{Ca}$ is the actual current flow capability of the branch; $\mathrm{Cn}$ is the actual current signature of the branch; Dr is the diagnosis outcome; Fun is the switch device attribute processing function.

The color set of Cy consists of all the switching branches; the color set of $\mathrm{Cc}$ is $\{0,1,2, x\}$, where $\{0\}$ means that this branch should be capable of conducting current backwords, $\{1\}$ means that this branch should be capable of conducting current forwards, $\{2\}$ means that this branch should be capable of conducting current in both directions, and $\{x\}$ means that the capability is still uncertain; the color set of $\mathrm{Cp}$ consists of all the ideal current signatures when the power 


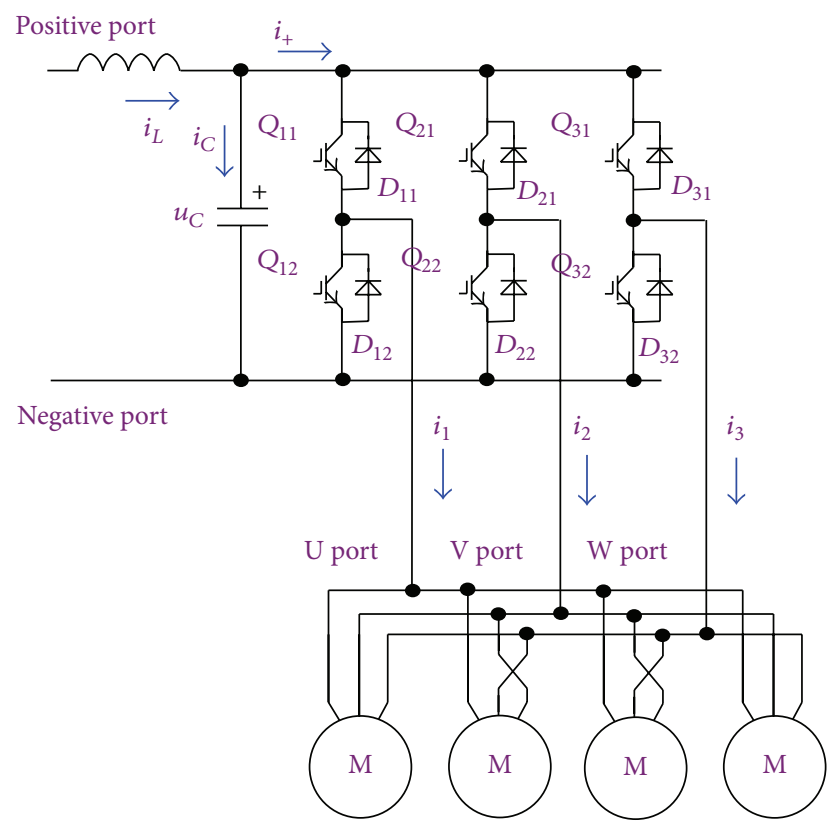

FIgURE 3: The power circuit layout of a traction converter in a rail transit vehicle.

circuit is fault-free; the color set of Cn consists of all the current signatures that can be detected and stored when the power circuit is with a broken switching device; the color set of $\mathrm{Ca}$ is $\{0,1,2, x\}$, while $\{0\}$ means that this branch has been detected to be capable of conducting current backwords, $\{1\}$ means that this branch has been detected to be capable of conducting current forwards, $\{2\}$ means that this branch has been detected to be capable of conducting current in both directions, and $\{x\}$ means that the actual capability is still uncertain;the color set of $\operatorname{Dr}$ is $\{0,1,2,3,4\}$, where $\{0\}$ means that this branch is operating normally, $\{1\}$ means that this branch has failed to conduct forward current while it is supposed to do so, $\{2\}$ means that this branch has failed to conduct backword current while it is supposed to do so, and $\{3\}$ means both $\{1\}$ and $\{2\}$ happen at the same time; the function attribute of Fun takes out the color in Cp, projects the color into $\mathrm{Cc}$, and then deletes the color, while it does the same to $\mathrm{Cn}$ and $\mathrm{Ca}$.

The definitions of all the transitions and places in the OOCPN reasoning machine are listed in Table 1.

\section{The Realization of Switching Circuit Topology Reasoning and Fault Diagnosis}

Generally, a power electronic switching topology with two inputs and three outputs is taken as an example here. Such topology is the well-known "full-bridge converter and rectifier," that is, the power circuit layout of a motor inverter with braking capability. In a motive car of rail transit vehicle, a motor inverter which is also known as traction converter (TC, shown in Figure 3) drives 4 parallel-connected traction motors to supply driving or braking force to the vehicle.

The realized process could be divided into the following 4 steps, as shown in Sections 3.1-3.4.

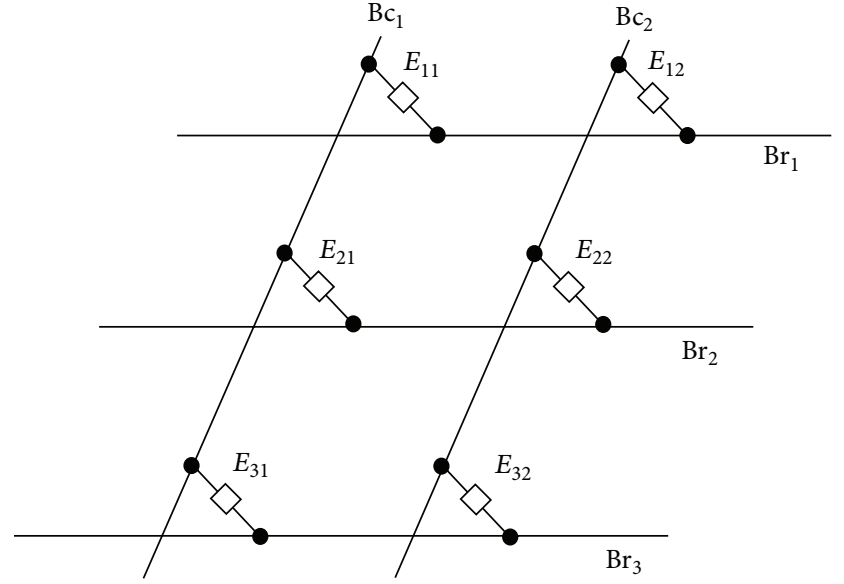

FIgURE 4: The simplified equivalent switching network of a traction converter in a rail transit vehicle.

3.1. To Simplify the Power Circuit into an Equivalent Switching Network. During the vehicle's traction stage, TC absorbs energy from the DC grid input (positive/negative port), converts the energy into three-phase $\mathrm{AC}$ power, and then supplies the AC power through three-phase AC outputs ( $U$, $V, W$ ports) to the motors; during the vehicle's braking stag, TC absorbs AC energy from the motors through AC outputs and feeds such energy back into DC grid inputs. The topology of a TC can be simplified into a 2 by 3 equivalent switching network, with 6 switching branches in all.

According to the layout in Figure 3, the power circuit of a TC is simplified into the equivalent network shown in Figure 4. In Figure 4, the $\mathrm{Bc}_{1}$ and $\mathrm{Bc}_{2}$ ports correspond to TC's positive and negative input ports, respectively; the $\mathrm{Br}_{1}$ $\mathrm{Br}_{3}$ ports correspond to TC's $U, V$, and $W$ output ports, respectively; $E_{11}, E_{12}, E_{21}, E_{22}, E_{31}$, and $E_{32}$ are the switching branches wiring input ports to output ports. As a matter of fact, $E_{i j}(i=1 \sim 3, j=1 \sim 2)$ consists of an IGBT of $Q_{i j}$ and an antiparalleled diode of $D_{i j}$.

\subsection{To Derive Coded Port Currents, for the Representation of Ideal and Actual Current/Voltage Signatures}

3.2.1. The Amplitude Coding of Currents through Input/Output Ports. Currents through positive $\left(i_{+}\right)$and negative $\left(i_{-}\right)$meet Kirchhoff's current law, that is,

$$
i_{+}-i_{-}=0 .
$$

According to (4), $i_{+}$and $i_{-}$are co-related; therefore the coded $i_{-}$may be ignored, considering such high coupling relationship. The ignorance of $i_{-}$will reduce the requirement for storage capacity of the reasoning machine by $50 \%$. However, although the relationship among $i_{1 \sim 3}$ could be derived by Kirchhoff's current law (as is shown in (5)), too, the ignoration of any one of them will increase the fault diagnosis time consumption greatly, because the AC current outputs effect more directly on actual currents that flow through the branches, and such ignorance implies more reasoning steps that must be taken by the OOCPN network. 


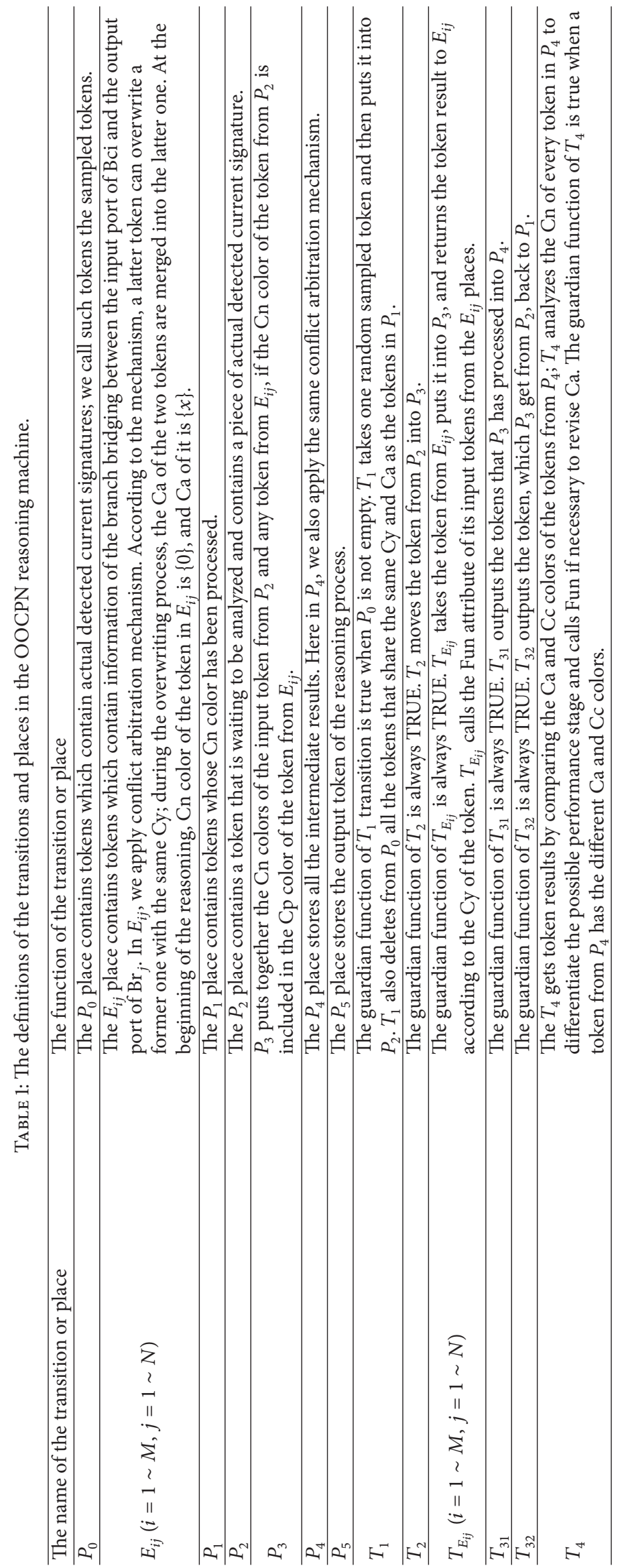


Through experiments, the time consumed by reasoning may be increased by an average of $30 \%$ in such case.

$$
i_{1}+i_{2}+i_{3}=0 \text {. }
$$
by

The digital coding of the currents $i_{+}, i_{1}, i_{2}, i_{3}$ is carried out

$$
\operatorname{sig}_{1}\left(\overline{i_{x}^{*}}\right)=\left\{\begin{array}{ll}
0, & \overline{i_{x}^{*}}<-\operatorname{thres}\left(\overline{i_{x}^{*}}\right) \\
1, & \overline{i_{x}^{*}}>\operatorname{thres}\left(\overline{i_{x}^{*}}\right),
\end{array} \quad x=+, 1,2,3 .\right.
$$

In (6), $i_{x}, x=+, 1,2,3$, are normalized with their nominal values, and the normalized values are then filtered by moving window approach. The filtering will eliminate all the highfrequency disturbance exerted by load surge/dive and by electromagnetic interference sources. $\overline{i_{x}^{*}}$ is the normalized mean value in (6). $\overline{i_{x}^{*}}$ is then compared with corresponding threshold thres $\left(\overline{i_{x}^{*}}\right)$, generating the current signature $\operatorname{sig}_{1}\left(\overline{i_{x}^{*}}\right)$. It should be noted that with a moving window average calculater the mean values are derived in such a way that helps to detect the variations of mean values much more quickly [18].

For TC, $i_{+}$is actually difficult to be detected owing to the existence of supporting capacitor. So $i_{+}$must be calculated or observed with the detectable inductor current $i_{L}$ and capacitor voltage $u_{c}$ :

$$
i_{+}^{*}(k)=\frac{i_{L}(k)-C\left[\left(u_{c}(k)-u_{c}(k-1)\right) / 2 T_{s}\right]}{i_{\text {nom }}} .
$$

In (4), the reconfigured $i_{+}$on time spot $k T_{s}\left(i_{+}^{*}(k)\right)$ is calculated with $i_{L}(k), u_{c}(k)$, and $u_{c}(k-1) . T_{s}$ is the sampling interval, $C$ is the capacitance of the supporting capacitor, and $i_{\text {nom }}$ is the nominal current adopted during the normalization. For a TC with the switching frequency of $1 \mathrm{kHz}, T_{s}$ should be no more than 0.2 milliseconds to ensure sufficient response bandwidth. Here we choose $T_{s}$ to be $100 \mu$ s.

There are 16 possible combinations of $\operatorname{sig}_{1}\left(\overline{i_{1}^{*}}\right) \sim \operatorname{sig}_{1}\left(\overline{i_{3}^{*}}\right)$. However, by (2), the combinations of $\{0,0,0,0\},\{1,0,0,0\}$, $\{0,1,1,1\}$, and $\{1,1,1,1\}$ will never exist in reality, so they are eliminated.

The determination of hysteresis band threshold thres $\left(\overline{i_{x}^{*}}\right)$ has serious effects on the accuracy of coding process when the current is around its zero value or relatively smaller. Here the thres $\left(\overline{i_{x}^{*}}\right)$ for TC is given as

$$
\text { thres }\left(i_{x}^{*}\right)= \begin{cases}0.10, & x=+ \\ 0.15, & x=1,2,3\end{cases}
$$

The coded current amplitudes are given as $\left\{\operatorname{sig}_{1}\left(\overline{i_{+}^{*}}\right)\right.$, $\left.\operatorname{sig}_{1}\left(\overline{i_{1}^{*}}\right), \operatorname{sig}_{1}\left(\overline{i_{2}^{*}}\right), \operatorname{sig}_{1}\left(\overline{i_{3}^{*}}\right)\right\}$. However, it is difficult to reason the circuit topology accurately merely with coded current amplitudes. For example, under the coded current amplitudes of $\{0,1,0,1\}$, there are two possible circuit layouts, as shown in Figure 5. In Figure 5, the device in the dashed box is represented to be "turned on." This is exactly the reason why the coding of current changing rates is necessary.
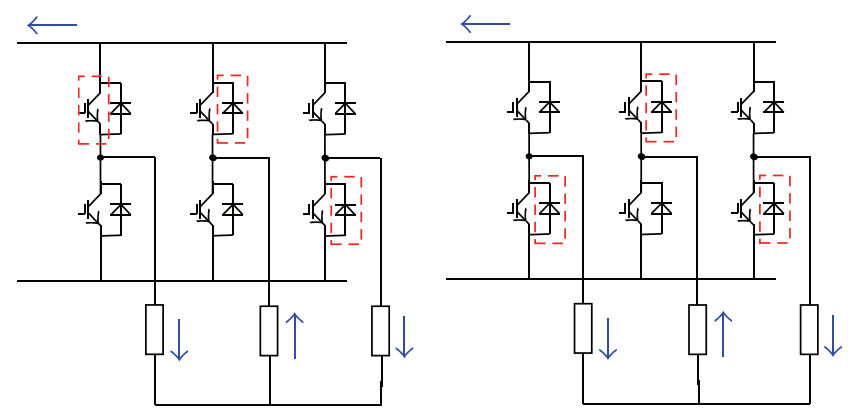

FIgURE 5: Possible circuit layouts under the coded current amplitudes of $\{0,1,0,1\}$.

\subsubsection{The Coding of Current Changing Rates through Output} Ports. The coding of current changing rates could be carried out as shown in

$$
\begin{aligned}
& \overline{i_{x}^{*}}(k)=\frac{\overline{i_{x}(k)}}{i_{\text {nom }}}, \\
& \frac{d}{d t} \overline{i_{x}^{*}}=\frac{\overline{i_{x}^{*}(k)}-\overline{i_{x}^{*}(k-1)}}{T_{s}} \\
& x=1,2,3, \\
& x=1,2,3 .
\end{aligned}
$$

Likewise, thres $\left(d / d t\left(\overline{i_{x}^{*}}\right)\right)$ here is selected to be 0.02 to ensure the accuracy.

Finally, the coded current signature is expressed as

$$
\begin{aligned}
& \left\{\operatorname{sig}_{1}\left(\overline{i_{+}^{*}}\right), \operatorname{sig}_{1}\left(\overline{i_{1}^{*}}\right), \operatorname{sig}_{1}\left(\overline{i_{2}^{*}}\right), \operatorname{sig}_{1}\left(\overline{i_{3}^{*}}\right), \operatorname{sig}_{2}\left(\frac{d}{d t} \overline{i_{1}^{*}}\right),\right. \\
& \left.\operatorname{sig}_{2}\left(\frac{d}{d t} \overline{i_{2}^{*}}\right), \operatorname{sig}_{2}\left(\frac{d}{d t} \overline{i_{3}^{*}}\right)\right\} .
\end{aligned}
$$

3.3. To Derive the Color Set of Cp and Cn. The color set of Cp and $\mathrm{Cn}$ could be derived by combining the coded amplitude with the coded changing rate of currents.

Take the $\mathrm{Cp}$ and $\mathrm{Cn}$ of $\mathrm{Cy}=E_{11}$ as an example; the color set is $\{0101100,0110100,1100111,1100101,1100110,1101111$, 1101110, 1110111, 1110101\}.

Figure 6 shows the corresponding topology to each element of the color set. The equivalent switching network corresponding to every layout in Figure 6 is shown in Figure 7.

3.4. To Diagnose for Malfunctioning Switching Device by Analyzing the Topology with OOCPN Reasoning Machine. Now we put the IGBT of $Q_{11}$ into malfuncion state. The breakdown of $Q_{11}$ means that $E_{11}$ is deprived of its forward current conducting capability. With OOCPN reasoning machine, the actual current conducting capability of $E_{11}$ could be analyzed, 


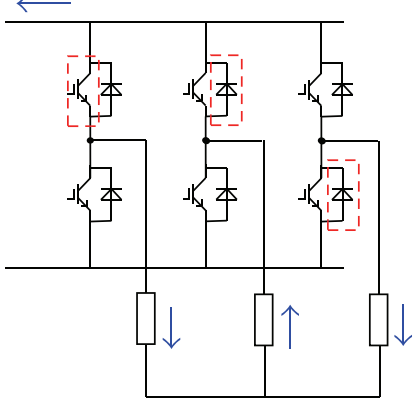

0101100

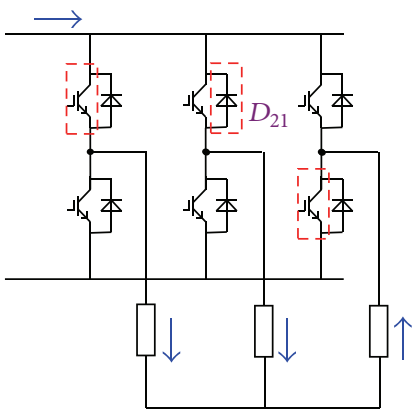

1100101

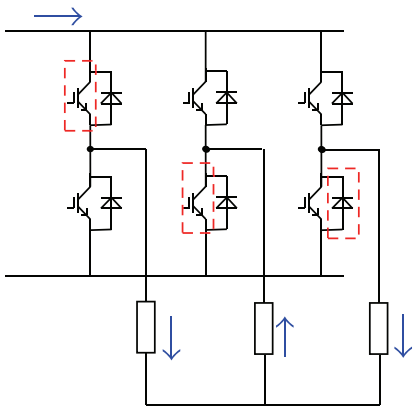

1101110

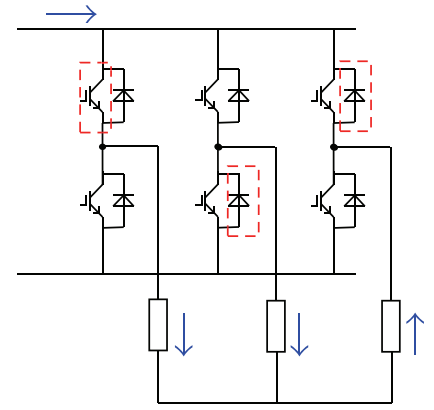

0110100

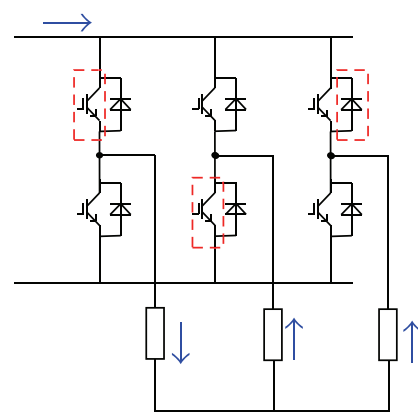

1100110

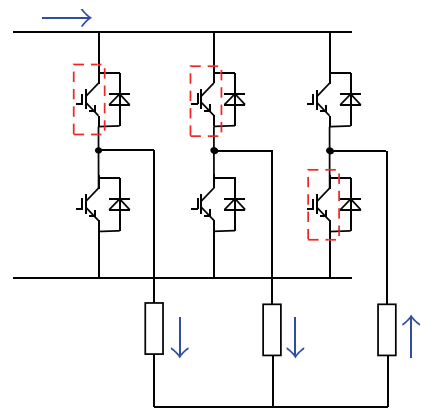

1110111

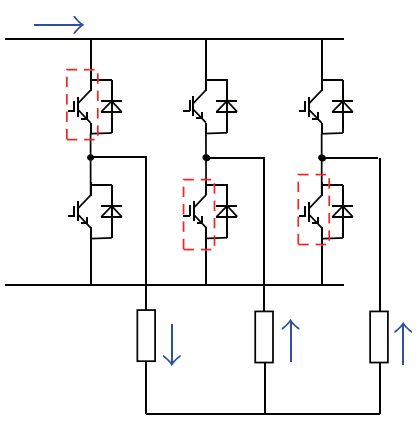

1100111

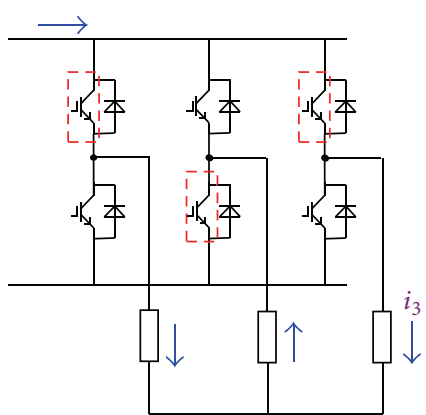

1101111

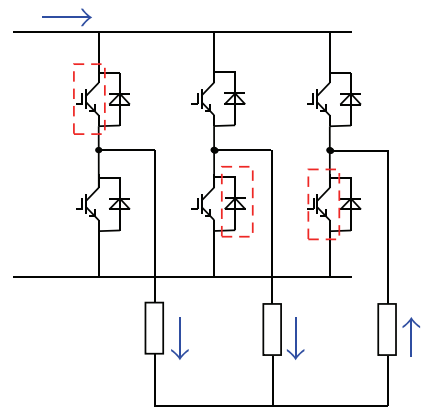

1110101

Figure 6: Possible circuit layouts corresponding to the color set of $\mathrm{Cp}$ and $\mathrm{Cn}$ with $\mathrm{Cy}=E_{11}$.

and it clearly means that $Q_{11}$ is being confronted with open circuit fault.

Before the reasoning process, the sampled current signatures are stored in the tokens in $P_{0}$. With a malfunctioning $Q_{11}$, all the colors of $\mathrm{Cn}$ in $P_{0}$ are as follows:

(1) During the traction stage:

\{1001111, 1001101, 0110011, 1010111, 1010110, 1010011, 1011111, 1011101, 1011110, 1101011, 1110011\}.

(2) During the braking stage:

\{0001000, 0001010, 0001100, 0010000, 0010001, 0010100, 0011000, 0011001, 0011010, 0100000, 0100001, 0100010, 0101000, 0101001, 0110000, 0110010\}.

After deriving the initial tokens, OOCPN runs freely according to its intrinsic rules. The major steps during reasoning are given in Table 2, under the circumstance of traction stage and malfunctioning $Q_{11}$. It should be noted that, in this example, $T_{4}$ differentiates the traction stage from the braking stage by the highest bit of $\mathrm{Cn}$ (i.e., $\left.\operatorname{sig}_{1}\left(\overline{i_{+}^{*}}\right)\right)$. After 137 steps, the token in place $P_{5}$ results in $\mathrm{Cy}=E_{11}$ and $\mathrm{Dr}=$ 1 , which means that $E_{11}$ is not capable of conducting forward current; that is, $Q_{11}$ is unable to be turned on.

In a prototype $\mathrm{TC}$ equipment, we realize the diagnosis example as is stated before. The equipment capacity is $230 \mathrm{kVA}$ with a traction motor of $190 \mathrm{~kW}$. The DC grid voltage is $1500 \mathrm{VDC}$, and the switching frequency is $1 \mathrm{kHz}$. The prototype TC is equipped with a diagnosis board which is based on TI's DSP2812 structure. In the DSP2812, the tokens of OOCPN are expressed and stored as several structures, and the transitions of OOCPN are realized with $\mathrm{C}$ language. The feasibility of OOCPN is that the programming of OOCPN could be carried out strictly and easily according to the network layout, and the reasoning steps could be fully and totally predictable, making it easier for one to check the program operation effect. Figure 8 gives the diagnosis results. 


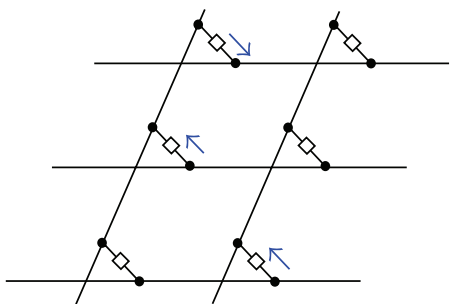

0101100

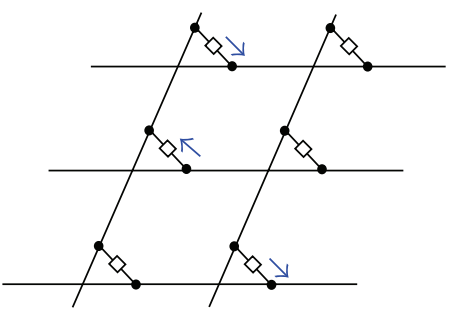

1100101

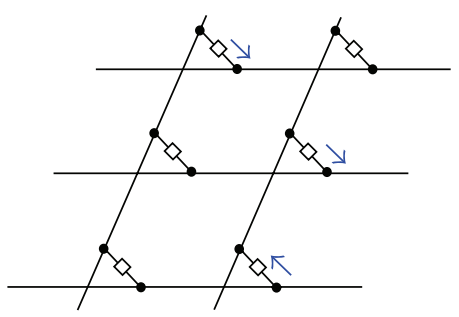

1101110

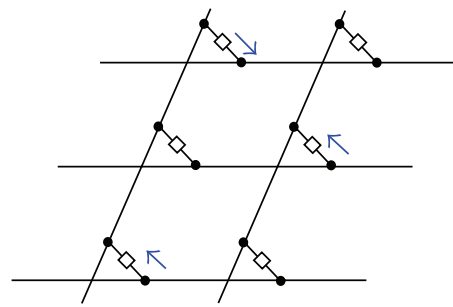

0110100

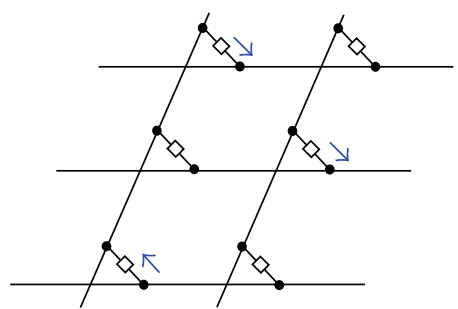

1100110

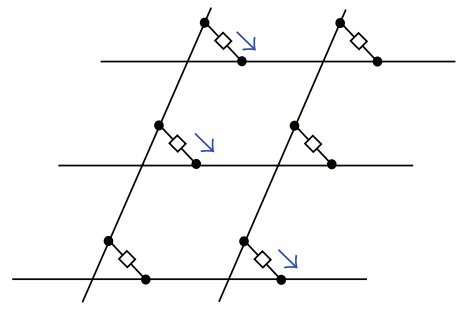

1110111

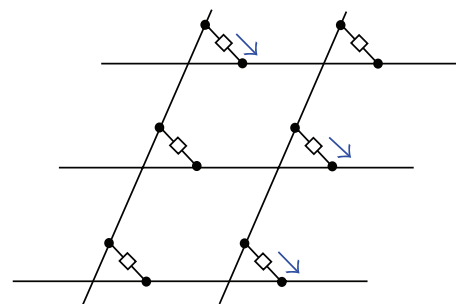

1100111

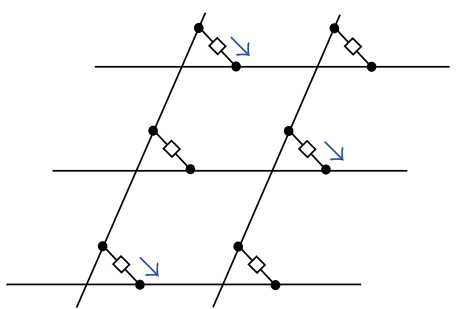

1101111

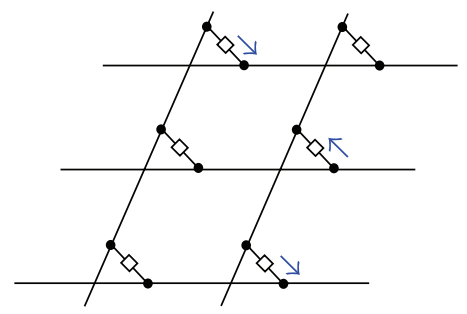

1110101

FIGURE 7: Possible equivalent switching networks corresponding to the color set of Cp and Cn with Cy $=E_{11}$.

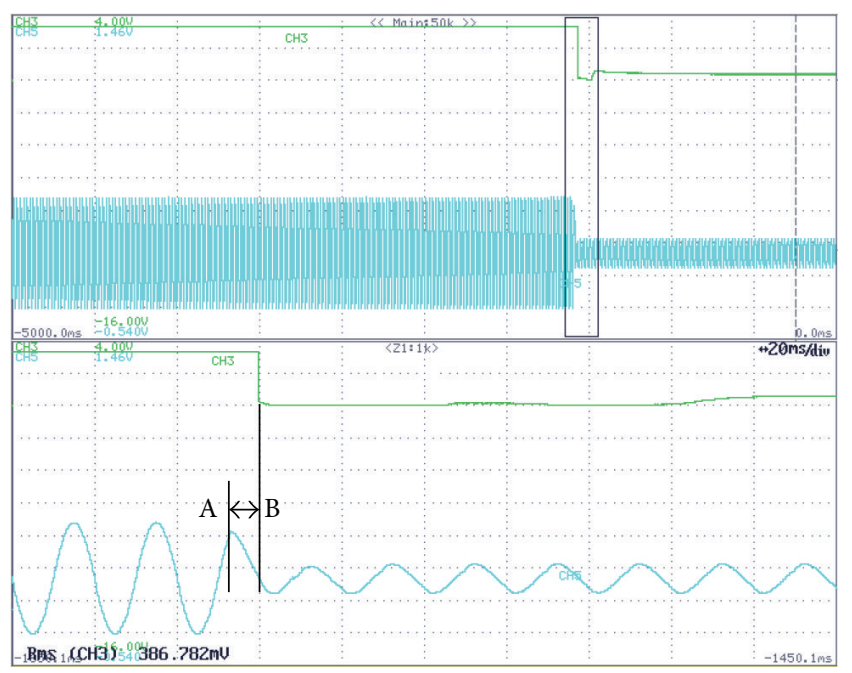

(a)

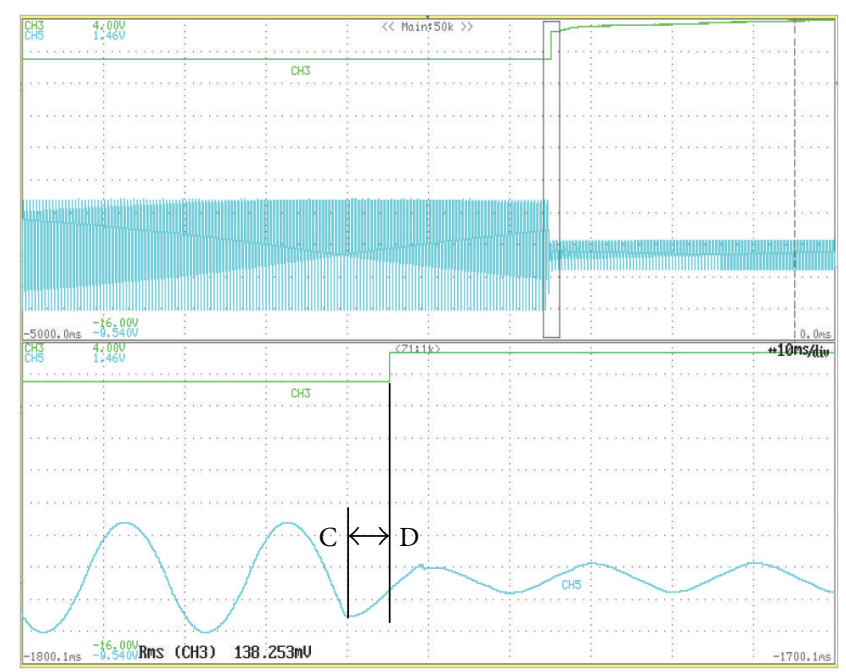

(b)

FIGURE 8: The waveform of $i_{1}$ and end mark of the fault diagnosis process with malfunctioning $Q_{11}$ in traction and braking stages. (a) In traction stage. (b) In braking stage. 
TABLE 2: During the traction stage of TC, the reasoning process of OOCPN model with malfunctioning Q11.

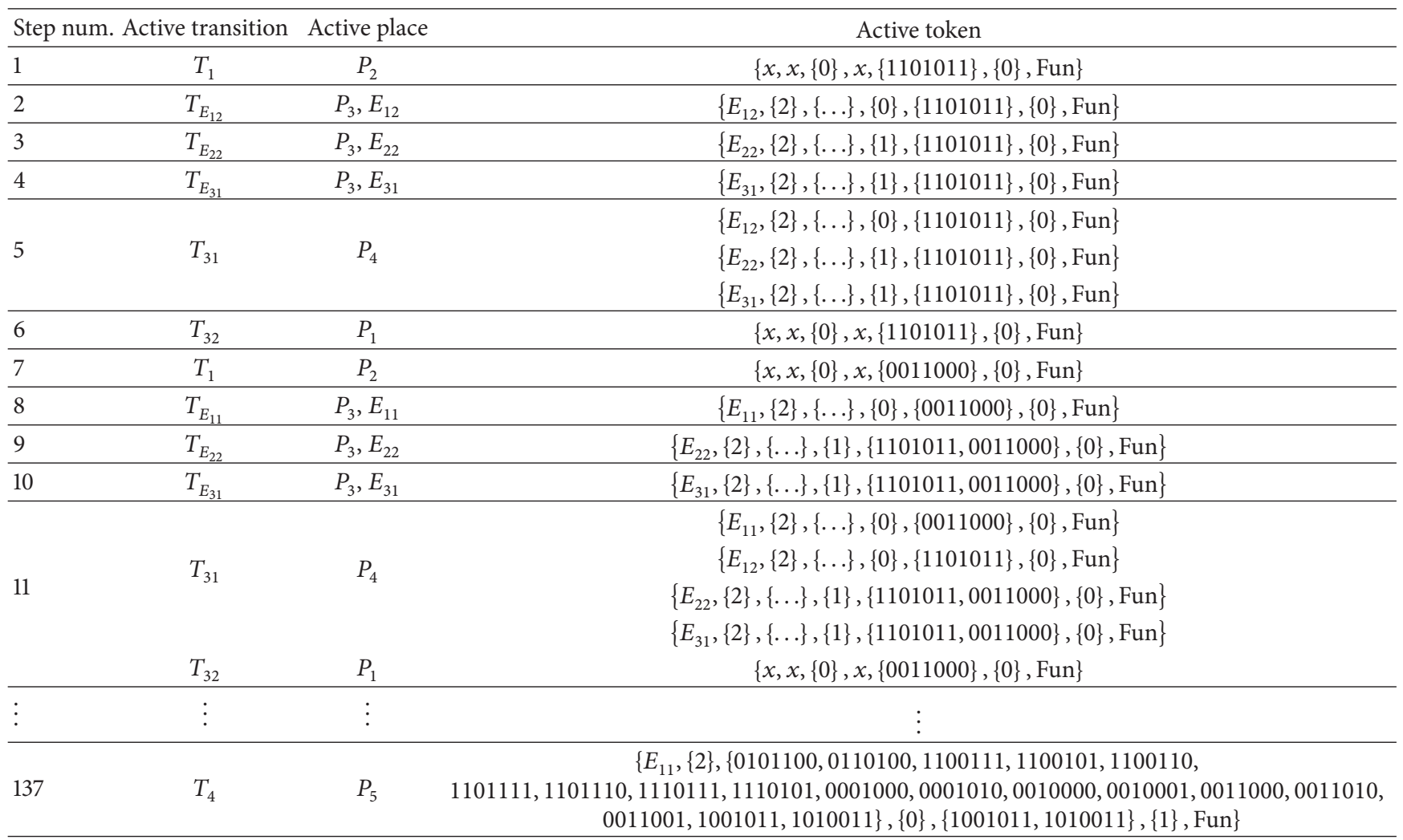

Figure 8 shows the waveform of $i_{1}$ and the waveform of end mark of the fault diagnosis process. Of the end mark, a rising edge means the fault happens during traction stage of TC, and a falling edge means braking stage. In both cases, the OOCPN model is run on a DSP 28335 platform from TI. Accurate malfunctioning device location is realized, with a maximum diagnosis delay of around $4.5 \mathrm{~ms}$. The diagnosis delay is defined as the time interval from the beginning of malfunctions to a rising or falling edge of diagnosis result. Such diagnosis delay is shown between time spots $A \sim B$ and $\mathrm{C} \sim \mathrm{D}$ in Figure 8. Such delay meets the real-time requirement in field application, which is usually less than one period time (typically 10 50 ms).

\section{Conclusions}

In the field of switching device open circuit fault diagnosis, an automatic reasoning machine based on object-oriented colored Petri net (OOCPN) is proposed in this paper. The proposed approach is related less to circuit parameters and simulates natural reasoning process carried out by an expert's brain. What is more, digitalized fault signatures accelerate the diagnosis process and offer higher disturbance-rejecting capability. Movement of the colored tokens, which are moved by transitions in an OOCPN, corresponds to the stream of consciousness and is easier to be realized in field applications. In our work, the application of OOCPN is key difficulty, and proper token definition finally makes it possible.

\section{Competing Interests}

The authors declare that they have no competing interests.

\section{Acknowledgments}

This work was supported by the Fundamental Research Funds for the Central Universities of China, no. E16JB00160/ 2016JBM062.

\section{References}

[1] R. L. De Araujo Ribeiro, C. B. Jacobina, E. R. C. Da Silva, and A. M. N. Lima, "Fault detection of open-switch damage in voltagefed PWM motor drive systems," IEEE Transactions on Power Electronics, vol. 18, no. 2, pp. 587-593, 2003.

[2] R. Peuget, S. Courtine, and J.-P. Rognon, "Fault detection and isolation on a pwm inverter by knowledge-based model," IEEE Transactions on Industry Applications, vol. 34, no. 6, pp. 13181326, 1998.

[3] Y. Hassaïne, B. Delourme, M. Sidorkiewicz, and E. Walter, "Identification of the topology of power system networks via mixed integer inequalities," in Proceedings of the IEEE Power Engineering Society General Meeting, pp. 199-204, June 2004.

[4] Y. Hassaine, E. Walter, M. Dancre, B. Delourme, and P. Panciatici, "Estimation of the topological state of power system networks via interval analysis," in Proceedings of the IEEE Power Tech Conference, pp. 6-8, Bologna, Italy, June 2003. 
[5] L. Mili, G. Steeno, F. Dobraca, and D. French, "A robust estimation method for topology error identification," IEEE Transactions on Power Systems, vol. 14, no. 4, pp. 1469-1476, 1999.

[6] Y. Shi, "Identification of isomorphic hybrid switching topology," Proceedings of the CSEE, vol. 23, no. 11, pp. 116-121, 2003.

[7] H.-L. Shang, Y. Liu, Z.-D. Liu, W.-J. Dong, and F. Li, "Application of optimized circuit simulation-identification of isomorphic hybrid switching," Journal of Applied Sciences, vol. 32, no. 2, pp. 199-208, 2014.

[8] H. Ma and Z.-X. Zhang, "Topology identification for power electronic circuits," Proceedings of the Chinese Society of Electrical Engineering, vol. 26, no. 6, pp. 55-60, 2006.

[9] Z.-X. Zhang, H. Ma, and X.-N. He, “Topology identification for power electronic circuits based on residual analysis," Proceedings of the Chinese Society of Electrical Engineering, vol. 26, no. 18, pp. 47-53, 2006.

[10] W. Zhang and Q. Y. Guo, "Fault diagnosis of the traction substation on the basis of petrinets technology," Urban Mass Transportation, vol. 7, no. 1, pp. 32-34, 2004.

[11] J. Sun, S. Y. Qin, and Y. H. Song, "Fuzzy petri nets and its application in the fault diagnosis of electric power systems," Proceedings of the CSEE, vol. 9, no. 24, pp. 74-79, 2004.

[12] J. Li, S. T. Wang, and L. P. Chen, "Simulation modeling based on the object-oriented Petri net for discrete event systems," Nature Science, vol. 29, no. 5, pp. 12-16, 2001.

[13] L. Xueshan, "The modeling and simulation environment based on object petri net for discrete event systems," Computer Simulation, vol. 17, no. 3, pp. 42-57, 2000.

[14] A. Jiaoyan, W. Zhenbiao, C. Dongzhen, and Y. Min, "Based on combination of object-oriented technology and colored-petri nets: FMS modeling," Journal of Hubei Polytechnic University, vol. 15, no. 1, pp. 14-17, 2000.

[15] T. Yewen, B. Yingcai, and D. Chenglin, "An object-oriented extension of colored petri nets," Mini-Micro Systems, vol. 20, no. 4, pp. 300-305, 1999.

[16] L. Qiongbo and Y. Jinyuan, "Application of coloured petri net in network communication protocol," Computer Engineering and Applications, vol. 9, pp. 27-46, 2001.

[17] X. P. Lai, H. X. Zhou, and L. Wang, "A coloured petri-net based algorithm for the topology analysis of power network," Control Theory and Applications, vol. 18, no. 5, pp. 726-731, 2004.

[18] L. Wang, Study on the Fault Diagnosis and Protection of EnergyFed Supply System in Urban Mass Transit, Jiaotong University, Beijing, China, 2010. 


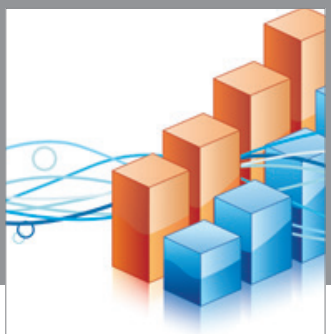

Advances in

Operations Research

vatem alat4

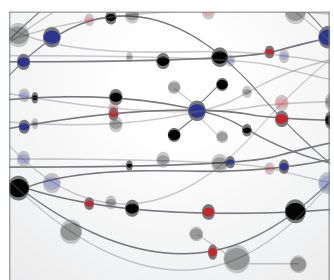

\section{The Scientific} World Journal
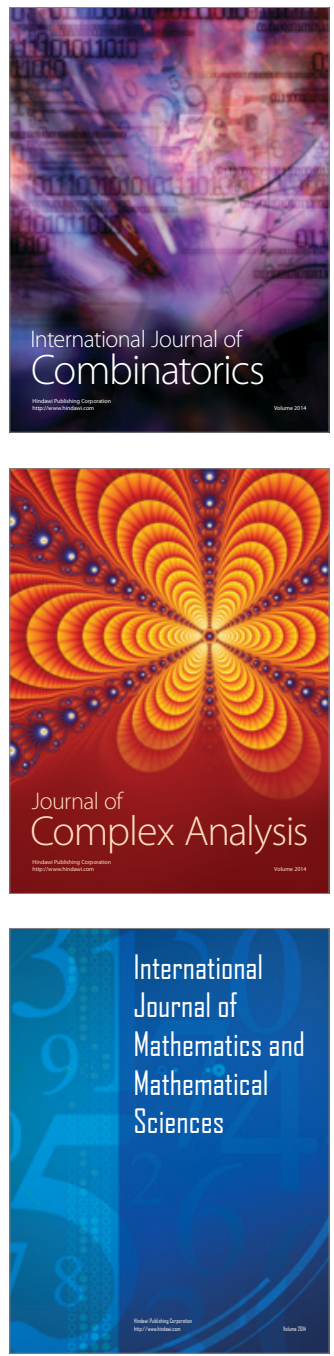
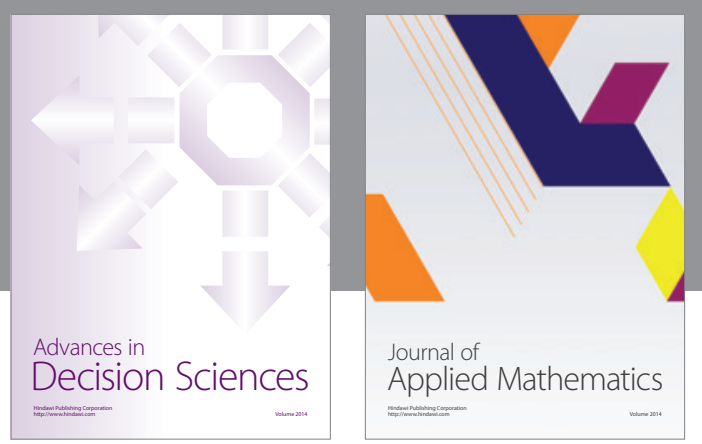

Algebra

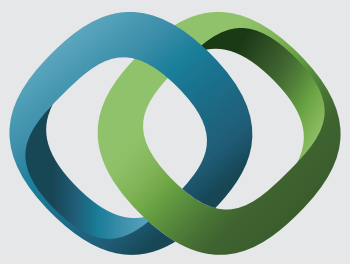

\section{Hindawi}

Submit your manuscripts at

http://www.hindawi.com
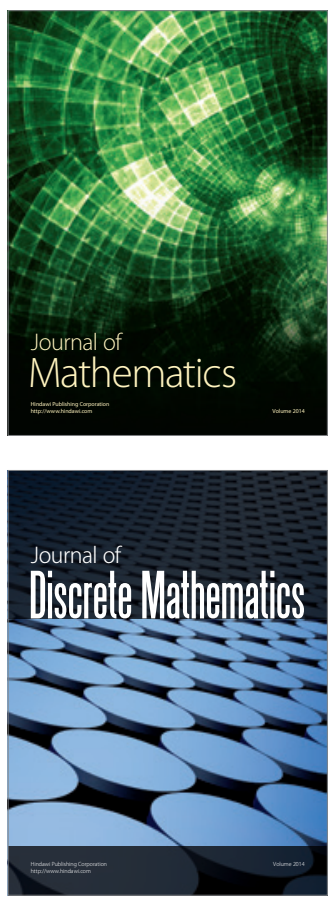

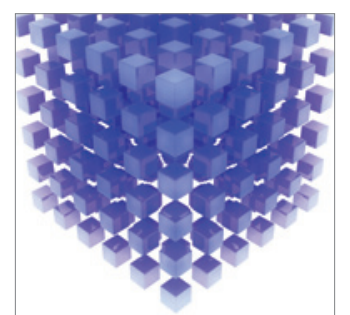

Mathematical Problems in Engineering
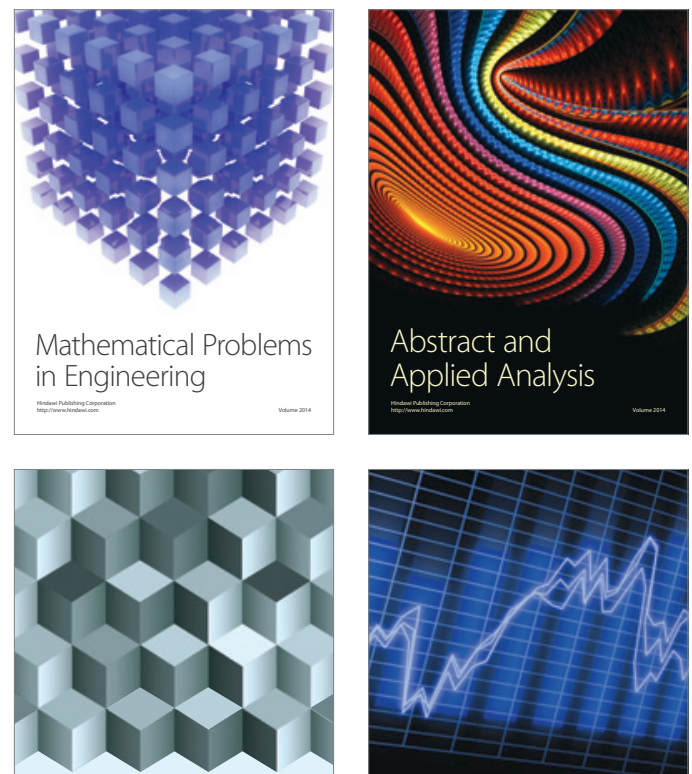

Journal of

Function Spaces

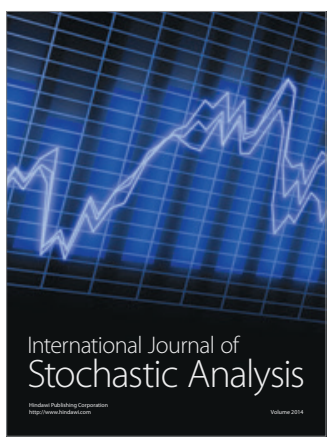

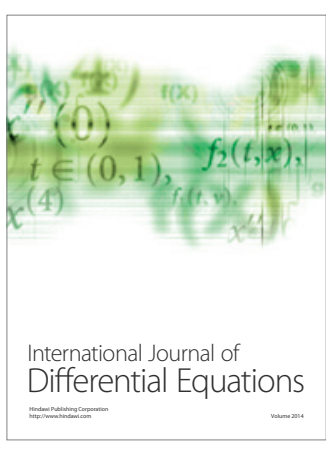
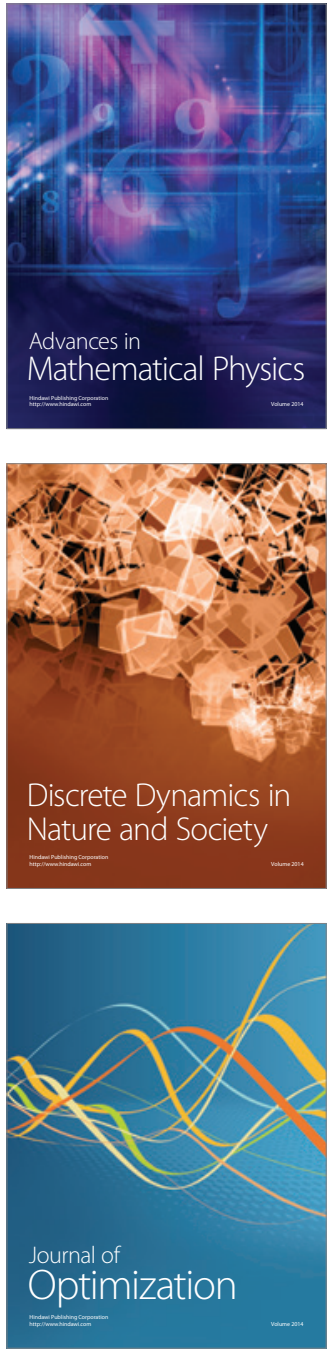